\title{
Spontaneous Four-Wave Mixing in an Irregular Nanofiber
}

\author{
A.A.Shukhin ${ }^{\text {a }}$ and A.A.Kalachev \\ Kazan E.K. Zavoisky Physical-Technical Institute, 420029, Sibirsky tract, 10/7, Kazan, Russia
}

\begin{abstract}
The features of biphotons states generated via spontaneous four-wave mixing in nanofibers with a variable cross-section are studied. The spectral amplitude of the biphoton field is calculated and the effects of interference and phase modulation of the biphoton field in such structures is discussed.
\end{abstract}

Keywords: spontaneous four-wave mixing, nanofiber, biphotons.

Currently, much attention is paid to basic research aimed at the development of quantum optical technologies [1]. In particular, coherent and nonlinear optical effects that are observed during the interaction of quantum states of light with nonlinear materials are of interest. Among them spontaneous parametric down-conversion and spontaneous four-wave mixing are used to create various one-photon and two-photon sources [2]. In general, devices that can create, store and convert the quantum states of light are the main elements of the optical quantum communication systems [3, 4] and optical quantum computers [5, 6]. In the future, such devices must ensure not only the efficiency and quality of processing of quantum states of light, but also miniaturization of optical schemes. From this point of view, optical nanofibers seem to be very promising objects.

Sub-micron diameter of fibers allows one to create small optical schemes, which are characterized by low loss and low energy consumption, and to efficiently bind conventional fibers and nanofibers (Fig. 1), which makes the optical schemes compatible with the integrated optics and nanophotonics. In addition, optical nanofibers enable a very simple way to make miniature high-quality cavities that have a wide range of applications in quantum optics. Therefore there is a need to study the basic features of nonlinear optical phenomena in such systems.

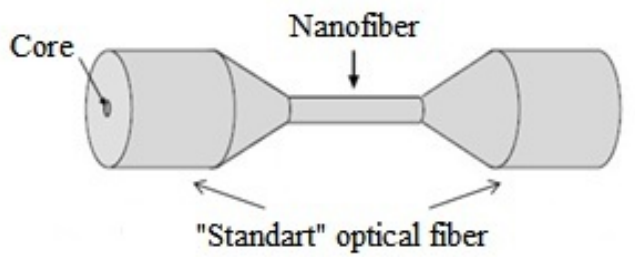

Figure 1. Illustrating a nanofiber as a tapered part of a standard optical fiber.

In the present work we develop the theory of spontaneous four-wave mixing (SFWM) in irregular nanofibers. We calculate the effective refractive index and mode functions for nanofibers that are

\footnotetext{
${ }^{a}$ Corresponding author: Spaun73@mail.ru
} 
made of various promising materials. The optimal size of a nanofiber for implementing phase matching condition allowing generation of correlated photons at wavelengths $810 \mathrm{~nm}$ and $1550 \mathrm{~nm}$ is determined. The spectrum of SFWM in irregular nanofibers is calculated and some interesting phenomena such as interference and phase modulation of the biphoton field, the description of which requires considering the effective refractive index as a function of the longitudinal coordinate, are discussed.

This work was supported by R’FBR (grants № 14-29-07192-ofi-m and 15-02-05478).

\section{References}

1. J.L. O’Brien, A. Furusawa, J. Vuckovic, Nature Photonics 3, 687 (2009)

2. M.D. Eisaman, et al., Rev. Sci. Instrum. 82, 071101 (2011)

3. N. Gisin, R. Thew, Nature photonics 1, 165 (2007)

4. N. Sangouard, et al., Rev. Mod. Phys. 83, 33 (2011)

5. P. Kok, et al., Rev. Mod. Phys. 79, 135 (2007)

6. T.D. Ladd, et al., Nature 464, 45 (2010)

7. Th.Knoche, Electronics Letters 32, 14, 1284-1285 (1996)

8. Q. Coulombier, Optics Express 18, 9, 9107-9112 (2010)

9. M. Kawachi, Electronics Letters 19, 15, 583-584(1983) 